can not, of course, be formed, and therefore restoration of function is not possible. As in sclerosis clsewhere the problem is to prevent further migration of leucocytes and to secure the maximum of efficiency from the remaining normal tissue.

Local applications in the form of sprays or vapors are certainly of benefit; medicines which act as mucous membrane stimulants, but not as irritants, are most efficient. Chloretone, many of the volatile oils, carbolic acid and iodin are most useful; mild alkalies, such as sodium benzoate and borate, are efficient in weak solu. tion for dislodging adherent mucus. All mineral astringents, especially silver nitrate, so long lauded in the treatment of chronic laryngitis, are out of place in this disease. and their use in any condition is exceedingly limited in the practice of the writer. The rather oldfashioned and now much-neglected steam atomizer is especially efficient, and is generally the only apparatus safe to trust to the hands of patients. Internal medication as far as the laryngo-tracheal disease is concerned is idle toil, not the slightest good ever having resulted from such treatment, used alone, under the writer's observation.

Massage, our most valuable agent when dealing with sclerosis higher up in the air passages, can scarcely be used even in the upper regions of the larynx, but may be cautiously attempted in very tolerant subjects. Faradism is of distinct value, the positive pole against the pharyngeal mucous membrane, and the negative over the sternum or held in the patient's hand, is the usual method of employment. A combination of external massage with faradization is very beneficial, one pole being held by the patient, the other by the surgeon, while with his other hand the latter carefully palpates the whole exterior of the larynx. Exercise of the upper respiratory organs by breathing and vocal exercises often does great good, and the improvement of the whole bodily muscular tone by properly directed ${ }^{*}$ general exercise is here. as in other forms of fibrosis, often singularly beneficial. Climatic change is always, if well directed, of use, sometimes arresting the sclerosis for a long period. A high altitude commonly answers best, and these cases are nearly always worse at the seashore.

The writer would urge greater frankness to ourselves as well as to our patients in dealing with these and other incurable cases, but believes that very great comfort, lasting for many years, can be secured in even some of the worst examples of chronic fibrosis of the air passages.

\section{THE PATHOLOGY THAT REMAINS AFTER THE NON-SURGICAL TREATMENT OF PERITONITIS.*}

H. D. NILES, M.D.

SURGFON TO THE HOLY CROSS HOSPITAL.

SALT LAKE CITY, UTAII.

Faithful followers of the teachings of rational medicine believe that actual disease within the abdomen, as in other parts of the body, always means a pathology, the removal or correction of which is essential to the restoration of normal health; and that the efficiency of any treatment for disease in this region, as elsewhere, should be estimated chiefly by the effect it has in removing or modifying the pathology which produces the symptoms. In the management of peritonitis, where the morbid processes are concealed beneath a rigid or

* Read at the twelfth annual meeting of the Western Surgical and Gynecological Association, held at St. Joseph, Mo., December 29 and $30,1902$. distended abdomen, there are many temptations to lure us from these teachings; and doubtless at times we all find ourselves reading. discussing, or perhaps practicing treatments which, in the light of the revelations of the modern operating-room, we must know are contrary to sound surgical principles and common sense.

I believe that we are especially prone to fall into these errors in our consideration of the remote dangers and distresses of peritonitis. Absorbed in our efforts to avert the immediate danger from sepsis, we are inclined to forget that the sufferings and deaths from recurrent attacks and adhesions are, in the aggregate, vastly greater than those occurring in primary attacks of acute peritonitis; and that the pathology remaining after the non-surgical treatment of peritonitis is not a myth, but a distinct, definite reality. which is directly responsible for this mortality and misery.

Bedside, laboratory and dead-house studies of this pathology and its causative relation to abdominal symptoms are instructive and interesting; but I believe that our most impressive and convincing lessons are to be learned in the operating-room, and that our perfected treatment of the future will be evolved from the gradually accumulated findings and conclusions of those who have frequent opportunities for observing these morbid processes in life.

The conclusions, briefly stated below and offered for your consideration, have been suggested or confirmed by my own operating-room observations. For the sake of brevity and clearness, these conclusions are compressed into the fewest possible words, free from statistics, case-notes, or quotations from authorities.

Ninety-five per cent. of all the survivors of the nonsurgical treatment of peritonitis are left with infection without the peritoneal cavity and adhesions within the peritoneal cavity. Well-known anatomic peculiarities of the gall bladder, appendix, and fallopian tubes favor the reception and imprisonment of infection from the alimentary canal and endometrium and its extension to the peritoneum. Consequently, it is about one of these organs that we usually find the pathology. unless it has been mechanically removed. The greatest amount of infection comes from a sudden rupture of an appendiceal or tubal abscess, or a perforation of the stomach, an intestine, or the gall bladder. The most virulent infection is either appendiceal or from a pyosaIpinx, following a puerperal endometritis; the mildest is from a cholangitis, or a gonorrheal salpingitis.

Since the infection of an appendicitis or a pyosalpinx has no outlet through natural channels, and in inflammation of the bile ducts and gall bladder there is usually some drainage through the common duct, we usually find in the first mentioned diseases the local and general symptoms are pronounced and unmistakable, while in the latter (in the absence of gallstone seizures) the symptoms may be very obscure and misleading. When the infection reaches the outer coat of one of these organs, peritoneal adhesions are formed as a part of $\mathrm{Na}$ ture's defense against the invasion of a microbic foc.

My own experience would lead me to believe that about 40 per cent. of the possessors of this pathology (infection and adhesions) suffer from recurrent attacks of acute and subacute peritonitis, and less than 1 per cent. from mechanical obstruction of the bowels.

It is to the remaining 59 per cent. that I invite your particular attention at this time; for, while it has been customary to point to these cases as sufficient proof of the efficiency of the drug treatment of peritonitis, I believe that all fair-minded, thoughtful observers are learn- 
ing to regard the pathology these patients carry within their abdomens as responsible for much distress and many deaths that we all formerly attributed to other causes, or were unable to trace to any well-defined cause.

It is difficult to conceive how this or any other pathology within the abdomen and outside of the alimentary canal can be removed or permanently modified by the application of drugs to the inner coat of the stomach or intestines or to the skin covering the abdomen; and, to my mind, it seems unreasonable to assume that the gall bladder, appendix, or the fallopian tube can continue to harbor infection and the patient be exempt from those general and local symptoms that accompany imprisoned infection in other parts of the body. Among the results that are commonly overlooked or misinterpreted are subacute local inflammations, mild systemic infection, chronic rheumatism affecting the joints, tendon sheaths, and, occasionally, the pleura, endocardium, and pericardium, and metastatic abscesses.

If we should make it a rule in examining every patient who has had peritonitis and apparently recovered without the mechanical removal of the primary cause, to drawn a line from the end of the tenth rib on the right side to the umbilicus, and another line from the anterior superior spine of the ilium to the umbilicus, and a third (if the patient is a female) from the middle of the second line to the spine of the pubes (each side), and then apply deep and direct pressure over the center of each of these lines, the existence and situation of a pathology would be, in nearly every instance, demonstrated. Such information would furnish us with an explanation of the symptoms of mild systemic infection, from which such patients often suffer. ${ }^{1}$

Just how much and how little general absorption may take place from a low-grade infection of the appendix, gall bladder or fallopian tube is impossible to determine, but that some lives are shortened in this way and many made miserable, is undoubtedly true. When we meet with patients suffering from symptoms that may be due to a mild toxemia of obscure origin, such as febrile and circulatory disturbances, gastro-intestinal disorders, headaches, fugitive pains, malaise, and others equally significant, we should always include in our examination those organs which are most likely to harbor the source of such a systemic infection.

For example, we know that rheumatism is often associated with appendicitis and peritonitis as well as with posterior urethritis; and, of late, we frequently hear of a rheumatic peritonitis or a rheumatic appendicitis, but never of a rheumatic gonorrhea. The fact is that rheumatism is not an uncommon consequence of septic absorption, and must be counted as one of the not infrequent remote results of the non-surgical treatment of peritonitis. It is surely inconsistent to conclude that infection in one part of the genito-urinary tract is a cause of rheumatism, and that in another or even in the gall bladder or appendix, it is the result of uricacidemia.

To perform their physiologic functions perfectly, the stomach and intestines require the greatest mobility their anatomic attachments permit, and any band of adhesion that fixes, binds, or drags on them or that impedes or prevents their free movements always means a departure from health, and is usually attended by more or less distress and danger.

Post-operative adhesions, however firm and strong, are usually absorbed if the primary infection is com-

1. I reported one such case in the March Annals of Surgery, where the infection came from a gall bladder filled with gallstones so situated as not to prođuce any characteristic paroxysms of pain. pletely removed; but no dietetic measures, drug treatment or rest cure will prevent the formation of adhesions or materially influence the absorption of those already formed, so long as the original infection is retained. In other words, the presence or absence of infection determines whether existing adhesions shall be absorbed or strengthened and multiplied. In operative work, the presence of adhesions about the gall bladder, appendix or fallopian tube is more reliable and tangible evidence of existing infection than any available microscopic or bacteriologic proof.

Guided alone by our observations during operations, I think that we may assume that either in the right upper quadrant or in one of the two lower quadrants of the abdomen, adhesions remain and produce symptoms more or less grave, in nine-tenths of all the cases after the non-surgical treatment of peritonitis. This pathology produces symptoms so variable in intensity and so misleading in character that unless one is impressed with its frequent occurrence and bears well in mind the anatomic relations and physiologic functions of the near-by organs, he is almost certain to attribute the symptoms to other and wrong causes.

Gastric symptoms are rarely absent, since the omentum is almost invariably involved in a way to drag on the stomach. Constriction about the pylorus with dilation of the stomach is not an infrequent result of gallbladder adhesions with a long list of gastric and reflex symptoms. In the majority of these cases, the true condition is not suspected, or, at least, demonstrated, since, unless life is in jeopardy, one does not often feel justified in opening the abdomen except the diagnosis be quite clear. Within the past few years, however, in a number of cases where the symptoms have been especially striking, the true diagnosis has been made, and the patient relieved by surgical measures. I have recently operated four times with satisfactory results, where the symptoms, produced by adhesions, pointed strongly to cancer or ulcer of the stomach.

Partial occlusion of the colon with or without a connecting sinus may occur when the gall bladder becomes attached to the hepatic curvature of the colon. ${ }^{2}$ Bands from diseased uterine appendages may encircle or angulate the ileum, when perhaps no history of recent pelvic disease exists to guide one. Involvement of the cecum and ileum are common after appendicitis and right pyosalpinx; while the sigmoid is nearly always implicated when the primary peritonitis has arisen from a left pyosalpinx.

It can not have escaped the observation of operators that ulcers of the stomach and intestines occur almost invariably in those parts that are most dependent or in those that are dragged on by adhesions. Doubtless longcontinued functional inactivity detracts from the vitality of the part and favors degenerative changes. It is equally true that the preferred site of malignant disease is about the former situation of an inflammatory process. Just as the cracked lip, the fissured nipple and the lacerated cervix seem essential to the development of cancer in those parts, so the selected regions for inflammation in the abdominal cavity seem to mark the favored sites of malignant disease, as the pylorus, duodenum, rectum, sigmoid, cecum, hepatic curvature, etc. If these facts be as true as operative experience would seem to indicate, we must count the pathology that remains after the non-surgical treatment of peritonitis as a

2. One case of my own of this kind was reported in the March Annals of Surgery. 
strong predisposing cause of ulcer and cancer, and in estimating the advantages and disadvantages of either method of treatment, due weight should be given these facts.

As in my recent work, I have compared the opinions formed at the bedside with the actual conditions disclosed on the operating table. It has seemed to me that we are only just beginning to comprehend the remote results that may follow the non-surgical treatment of peritonitis and that no itemized list of symptons produced by this infection and these adhesions will serve to give us an adequate idea of this pathology and its ill consequences; but that our clearest conception must come from a realizing sense of its relative frequency, as compared with other morbid conditions within the abdomen, the history of one or more attacks of peritonitis, the results of palpation, and the exclusion of other causative factors, combined with our familiarity with the anatomy and physiology of the parts involved.

Beyond the reach of drugs, electricity, massage, or dietetic measures, more common than cancer and ulcer combined, often mistaken for either, a predisposing cause of both, capable of producing any abdominal symptom or set of symptoms in the category from those of the mildest functional disturbance to those of welladvanced malignant disease, surely we have here a pathology that needs to be taken into the strictest account not only by surgeons who undertake the rational treatment of acute peritonitis, and are often called on to decide for or against operative interference, but by all of us when we are striving to search out the primary causative factors and associated symptoms of any obscure chronic disease or distress within the abdomen.

\section{THE USE OF A MYDRIATIC AFTER THE AGE OF FORTY-FIVE.* HORACE M. STARKEY. CHICAGO.}

In discussing this topic it will be well to keep in mind the distinction between mydriatics and cycloplegics, remembering that the former, in so far as they are mydriatics only, dilate the pupil without affecting the ciliary muscle, and that the effect desired in the latter is to suspend the action of the ciliary muscle and so paralyze the accommodation. While there is no known eycloplegic which does not at the same time dilate the pupil, there are mydriatics which have but little effect on the accommodation.

It is probable that our committee, in arranging the subjects for special discussion at this time, had more particular reference to the use of a cycloplegic in refraction work after the age of 45 , but the writer has had no intimation that this is the case, and the topic will receive a somewhat broader treatment. While the main discussion will center about such use of cycloplegics, the use of both mydriatics and cycloplegics for various purposes after middle life will be briefly considered.

\section{THE USE OF A MYDRIATIC.}

$a$ For ophthalmoscopy and $b$ for retinoscopy.

$a$ Concerning the use of a mydriatic for making more thorough examination of the peripheral portions of the lens, the other media and the. fundus there is perhaps no difference of opinion. We all probably use a mydriatic for this purpose at any age without the slightest

* Rear at the Fifty-thlrd Annual Meeting of the American Medical Association, in the Section on Ophthalmology, and approved for publieation by the Executive Committee: Drs. Frank Allport,
H. V. Wirdemann and J. A. Lipplncott. hesitation, unless we find symptoms of glaucoma present. In the latter case even we may consider the additional information gained of sufficient importance to warrant the use of a mydriatic for a sufficient time to make an examination and then follow it with a myotic, which shall counteract any unpleasant effect that might follow dilatation of the pupil.

$b$ The same rule of action probably governs the use of mydriatics in making the shadow test to determine the refraction. 'This is, in the writer's judgment, by far the most valuable objective test for refraction at our command, and yet in many cases, particularly in old people in whom the pupil is apt to be small, its accurate application without artificial mydriasis is impossible. In such cases, with the precautions mentioned above, a mydriatic is indicated.

NECESSITY OF A CYCLOPLEGIO IN CERTAIN CASES BETOND THE AGE OF 45 FOR ACCURATE REFRACTION.

On this point one would expect to find differences of opinion, as there are differences of opinion concerning the necessity of using a cycloplegic for refraction work at any age. To test their theory and practice in this regard the writer sent one hundred letters of inquiry to prominent ophthalmologists throughout the country. The number and promptness of the replies and the interest shown in the subject are most gratifying.* 'To those who so kindly contributed to this paper the writer wishes at this time to express his sincere thanks.

The writer has for many years believed that better and more accurate refraction work can be done for most patients under 30 by putting the accommodation entirely at rest at some time during the examination, and it is his practice to urge this procedure in all but the most simple cases. Beyond the age of 30 , the proportion for whom a cycloplegic is required rapidly diminishes, and by the time 45 is reached such cases are exceptional: and yet in the exceptional cases the indication is as positive for cycloplegia as in those of any age. Since the suspension of accommodation in the aged is due to hardening of the lens and not to paralysis of the ciliary muscle, it is difficult to fix a time beyond which a cycloplegic could never be of advantage. We occasionally find the ciliary muscles of eyes that have lost practically all accommodation still tugging away in an effort to correct an annoying astigmatism, and the suspension of the function of these muscles will sometimes disclose conditions not otherwise discoverable.

The writer did not suppose that a cycloplegic is used in more than 1 or 2 per cent. of his refraction cases after the age of 45 , but on running back over his records he finds that of 194 such cases seen in private practice in the past two years a mydriatic was used in 38 cases and a cycloplegic in 14 . Of the latter scopolamin was the cycloplegic employed in 9 cases, none over 50 years old, while homatropin and cocain were used in 5 cases, one of which was 55. Homatropin and cocain were used in one patient aged 50, and as the accommodation was not entirely suspended scopolamin was employed later. That there are such cases that can not be as well corrected with all available tests without cycloplegia he is fully assured. It is possible that if patients could be seen and studied repeatedly that proper correction might

* Since we all like to have others think as we do, it is an addi tional source of gratification to know that the practice of so large a proportion of those replying coincides as closely as it does witb that of the writer. The abstract of this paper [in the program of the Saratoga meeting] was sent to the Editor of THE JournaL before those answers were received, but a very large proportion of them show a practice almost identical with that outlined in that abstract. 